\title{
Cardiomiopatía diabética
}

\author{
Dr. José Aníbal Manfredi Carabetti
}

\section{Resumen}

La enfermedad coronaria, la hipertensión arterial y la diabetes son factores de riesgo independientes para el desarrollo de insuficiencia cardíaca y muerte. La cardiomiopatía diabética (CMD) es una de las etiologías frecuentes de cardiopatía en pacientes con diabetes tipo 1 y tipo 2. Si bien se suele plantear la CMD como la causa de la cardiopatía cuando se excluyen la hipertensión arterial, las valvulopatías y la enfermedad arterial coronaria aterotrombótica, estas coexisten con frecuencia e incluso también con la neuropatía autónoma cardiovascular. En los pacientes con CMD se puede demostrar mediante tests serológicos y por imagen alteraciones a nivel molecular, metabólico, mitocondrial, celular y tisular con infiltración grasa del músculo cardíaco, vinculadas a hiperglicemia, hiperinsulinemia y resistencia a la insulina, así como a lipotoxicidad por ácidos grasos libres, que son responsables del desarrollo de la CMD. Esta entidad primero determina disfunción diastólica del ventrículo izquierdo, más tarde disfunción sistólica e insuficiencia cardíaca. Se diagnostica mediante estudios serológicos de marcadores biológicos múltiples y por técnicas de imagen que evidencian la disfunción ventricular y mejoran la predicción pronóstica de enfermedad cardiovascular en diabéticos. En base a dichas pruebas se ha propuesto una clasificación por estadios o fenotipos clínicos de la CMD, que apunta a su diagnóstico precoz. Puede ser asintomática o ser responsable de síntomas y manifestaciones severas tales como insuficiencia cardíaca, arritmias y muerte súbita. Se puede asociar a hipertensión arterial, a enfermedad coronaria, a otras manifestaciones de microangiopatía y macroangiopatía aterotrombótica y a mortalidad cardiovascular. La prevención y el tratamiento intensivo multifactorial y personalizado de la diabetes, de todas sus alteraciones metabólicas y de la cardiopatía, mejoran la calidad y prolongan la vida. Se espera que investigaciones recientes, en proceso y futuras, determinen portentosos avances en la prevención y en el tratamiento de la CMD, que constituye una de las serias amenazas a la salud de la humanidad.

Palabras clave:

DIABETES MELLITUS

CARDIOMIOPATÍAS DIABÉTICAS/PREVENCIÓN \& CONTROL

CARDIOMIOPATÍAS DIABÉTICAS/TERAPIA

SIGNOS Y SÍNTOMAS

TÉCNICAS Y PROCEDIMIENTOS DIAGNÓSTICOS

\section{Diabetic cardiomyopathy}

\section{Summary}

Coronary heart disease, hypertension and diabetes mellitus are independent risk factors for heart failure and death. Diabetic cardiomyopathy (DCM) is one of the common etiologies of cardiac disease in patients with diabetes type 1 or 2 . Although DCM is often considered as the cause of heart disease when arterial hypertension, valvulopathies and atherothrombotic coronary artery are excluded, they coexist frequently, as well as with cardiovascular neuropathy. In patients with DCM, serological and imaging tests can show alterations at the molecular, metabolic, mitochondrial, cellular and tissue levels with fatty infiltration of the heart muscle, linked to hyperglycemia, hyperinsulinemia, insulin resistance, and lipotoxicity by fatty free acids, which are responsible for the development of the cardiomyopathy. The DCM first determines left ventricular diastolic dysfunction, later systolic dysfunction and heart failure. It is diagnosed by serological tests of multiple biological markers and by imaging tests that demonstrate ventricular dysfunction and improve the prognostic prediction of cardiovascular disease in diabetics. Based on these tests, a classification by stages or clinical phenotypes of DCM, which aims at its early diagnosis, has been proposed. It can be asymptomatic or be responsible for symptoms and severe manifestations such as heart failure, arrhythmias and sudden death, and may associate hypertension, coronary disease, other manifestations of microangiopathy and atherothrombotic macroangiopathy and cardiovascular mortality. The prevention and intensive multifactorial and personalized treatment of diabetes and all its metabolic and cardiac alterations, improve quality and prolong life. It is expected that ongoing and future research will determine breakthroughs in the prevention and treatment of DCM, which is one of the serious threats to the health of mankind.

Key words:

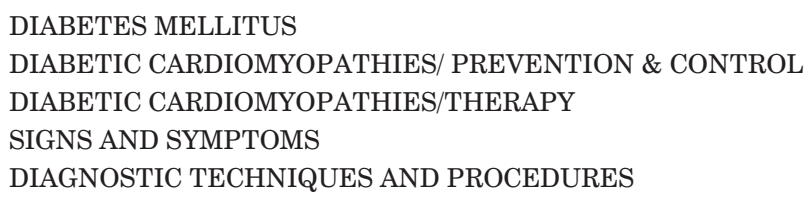

Médico Cardiólogo. Casa de Galicia. Instituto de Cardiología Integral. Clínica Pisabarro.

Correspondencia: Dr. Aníbal Manfredi. Correo electrónico: joseanibalmanfredi@yahoo.com

Recibido Ago 18, 2017; aceptado Oct 19, 2017 


\section{Introducción}

La causa primaria de mortalidad en pacientes con diabetes es la enfermedad cardiovascular, que corresponde a entre $50 \%$ y $80 \%$ de las muertes ${ }^{(1)}$.

La prevalencia de la diabetes en el mundo se está incrementando ${ }^{(2)}$ en adultos desde $4,7 \%$ en 1980 a 8,5\% en 2014 , y alcanzó a $12,4 \%$ de la población adulta de 35 a 74 años en el estudio basal en los años 2010 a 2011 de muestras seleccionadas al azar de cuatro ciudades del Cono Sur de América Latina: Bariloche y Marcos Paz en Argentina, Temuco en Chile y Pando-Barros Blancos en Uruguay ${ }^{(3)}$.

La enfermedad coronaria, la hipertensión arterial y la diabetes son factores de riesgo independientes para el desarrollo de insuficiencia cardíaca ${ }^{(4)}$.

Se prevé que la incidencia de insuficiencia cardíaca aumente en países en los que aumenta la edad de la población ${ }^{(5)}$, en países en desarrollo ${ }^{(6,7)}$, y en relación con los hábitos de vida no saludables, la supervivencia vinculada a mejores tratamientos de pacientes con cardiopatías ${ }^{(8)}$ y la concomitancia de comorbilidades, en especial la diabetes ${ }^{(9)}$.

La incidencia de insuficiencia cardíaca en personas con diabetes es cuatro veces mayor en hombres $\mathrm{y}$ siete veces mayor en mujeres que en personas sin diabetes $^{(9)}$.

Hasta $50 \%$ de las personas con diabetes tipo 2 pueden desarrollar insuficiencia cardíaca(4).

La concomitancia de diabetes en pacientes con insuficiencia cardíaca se asocia a alta mortalidad. La incidencia de internación por insuficiencia cardíaca y el riesgo de muerte se duplican y el de muerte súbita cardíaca se triplica con respecto a los no diabéticos ${ }^{(9,10)}$.

La diabetes se asocia a una alta incidencia de insuficiencia cardíaca congestiva y mayor pérdida de función contráctil cardíaca para el mismo tamaño del infarto miocárdico, contribuyendo a mayor discapacidad y mortalidad ${ }^{(11,12)}$.

La edad de aparición y la duración de la diabetes, así como el estado funcional, son predictores de mortalidad excesiva ${ }^{(9,10)}$.

La incidencia de cardiopatía en pacientes con diabetes tipo 1 y tipo 2 es alta, y si bien se incrementa con los años de diabetes, puede estar ya presente cuando se detecta clínicamente la diabetes tipo 2 .

La diabetes mellitus se relaciona con una alta incidencia de mortalidad en pacientes con cardiomiopatía isquémica aterosclerótica. En el estudio SOLVD, de portadores de insuficiencia cardíaca con fallo sistólico, el pronóstico adverso asociado a la etiología isquémica aterosclerótica de la cardiopatía se limitó a los que asociaban diabetes mellitus ${ }^{(13)}$. Esta sinergia deletérea puede tener relación con que en la cardiomiopatía isquémica hay un desvío desde la utilización de los ácidos grasos libres como principal sustrato miocárdico hacia la mayor dependencia de la glucosa y la glucolisis ${ }^{(13,14)}$, y que la captación de glucosa por las células musculares cardíacas requiere de insulina ${ }^{(15)}$ y está comprometida en la diabetes tipo $2^{(16)}$.

Incluso en ausencia de manifiesta diabetes mellitus, hasta dos tercios de los pacientes con insuficiencia cardíaca presentan resistencia a la insulina ${ }^{(17)}$, que no solo incrementa el riesgo subsecuente de diabetes mellitus, sino que también predice un pobre pronóstico(18).

\section{Cardiomiopatía diabética. Definición}

El desarrollo de insuficiencia cardíaca en personas con diabetes, que es independiente de la severidad de la enfermedad arterial coronaria ${ }^{(9,10)}$, de la edad y de la función renal ${ }^{(10)}$ y que se asocia a mayor requerimiento diurético, peor capacidad funcional y mayor riesgo de muerte cardiovascular(10), permite plantear que un proceso miocárdico es el mediador, mediante glucotoxicidad, disfunción microvascular y depósito anormal de colágeno ${ }^{(11,12)}$.

La CMD se ha definido como la disfunción miocárdica en pacientes con diabetes mellitus, en ausencia de hipertensión y de enfermedades cardíacas estructurales, como valvulopatías y enfermedad arterial coronaria ${ }^{(14)}$, y mejor aún como la enfermedad miocárdica en personas con diabetes que no puede ser atribuida al efecto individual de la enfermedad arterial coronaria, hipertensión u otra enfermedad cardíaca conocida ${ }^{(19)}$

La CMD es una de las etiologías de cardiopatía en pacientes con diabetes tipo $1^{(10,15,20)}$ y tipo $2^{(15,16,20)}$.

La diabetes tipo 1, aun en ausencia de cualquier otra enfermedad cardiovascular, puede conducir a disfunción ventricular y a CMD clínica, lo que fue comunicado por primera vez por Rubler en $1972^{(15)}$.

La diabetes tipo 2 se asocia a un incremento de la masa del ventrículo izquierdo y de su grosor parietal, y a reducción de su función sistólica y particularmente a disfunción diastólica, independiente de la hipertensión arterial(16).

Se ha estimado la prevalencia de la CMD en $12 \%$ de las personas con diabetes mellitus ${ }^{(15)}$.

Si bien se plantea la CMD como la causa de la cardiopatía cuando se excluyen, entre otras, la hipertensión arterial y la enfermedad coronaria, pueden coexistir e incluso también con la neuropatía autónoma cardiovascular ${ }^{(21)}$. 
Mecanismos fisiopatológicos de la cardiomiopatía diabética

En modelos experimentales animales y en personas con diabetes se han demostrado diversos cambios metabólicos, moleculares, funcionales y estructurales del miocardio que ocurren en el desarrollo de la $\mathrm{CMD}^{(22,23)}$.

El continuo y gran desarrollo de la medicina molecular se hace evidente al revisar la literatura científica sobre los estudios de los mecanismos fisiopatológicos en la CMD. La presente revisión solo pretende presentar algunos de los factores y procesos que se señalan como involucrados de acuerdo al conocimiento actual.

La incapacidad del miocardio para utilizar eficientemente los sustratos (lípidos o carbohidratos) subyace a la $\mathrm{CMD}^{(24)}$. En reposo el sustrato preferido del miocardio son los ácidos grasos libres y, con el ejercicio, el miocardio sano cambia al utilizar carbohidratos, los cuales generan más ATP para un monto dado de oxígeno ${ }^{(24)}$. A este cambio se le denomina flexibilidad metabólica, que en la diabetes está disminuida, lo que resulta en incremento de la incompleta oxidación de los ácidos grasos y en producción de aldehídos y especies reactivas de oxígeno, las que son tóxicas para el miocardio ${ }^{(24-26)}$.

Se ha sugerido que la resistencia a la insulina y la limitada perfusión del miocardio contribuyen a la disminución de la flexibilidad metabólica miocárdica y a la progresión de la $\mathrm{CMD}^{(24)}$.

La captación de glucosa por las células musculares cardíacas requiere de insulina ${ }^{(19)}$ y está comprometida en la diabetes tipo $2^{(20)}$ por la resistencia a la insulina. El compromiso de la señalización de la insulina juega un rol crítico en el desarrollo de la CMD. Los niveles altos de glucosa y lípidos generan aumento del estrés oxidativo, manejo defectuoso del calcio, alteración de la función mitocondrial, inflamación y fibrosis ${ }^{(24,26,27)}$.

La hiperglicemia y la resistencia a la insulina, con desvío del metabolismo de la glucosa hacia el incremento de la beta-oxidación y al consecutivo daño miocárdico por ácidos grasos libres -lipotoxicidad-activación del sistema renina-angiotensina-aldosterona, alteración de la homeostasis del calcio y cambios estructurales de la red de colágeno hacia una matriz más rígida con hipertrofia y fibrosis por la formación de productos finales de glicosilación avanzada, contribuyen a los diversos fenotipos clínicos de $\mathrm{CMD}^{(20)}$.

El estrés oxidativo aumentado o desequilibrio entre la producción de especies reactivas de oxígeno y su degradación por antioxidantes endógenos ha estado implicado tanto en el inicio como en la progresión de la insuficiencia cardíaca congestiva y de la disfunción cardíaca de la $\mathrm{CMD}^{(24)}$.
El persistente exceso de especies reactivas de oxígeno conduce a inflamación y fibrosis miocárdica ${ }^{(1,29)}$, como lo hace en la adventicia de las paredes vasculares arteriales, donde determina mayor rigidez arterial, que es otro factor de disfunción cardíaca y es frecuente en la hipertensión arterial. La rigidez arterial aumentada predice mortalidad.

La CMD se acompaña de alteración de la relación simpático/parasimpático, esto es, de neuropatía autónoma cardiovascular. Se señala un rol importante de la neuropatía autónoma cardiovascular en el desarrollo de la $\mathrm{CMD}^{(25,26)}$.

La CMD se caracteriza por la acumulación de lípidos en las células musculares cardíacas, la reactivación de genes fetales y la hipertrofia ventricular izquierda, alteraciones que en conjunto desembocan en la disfunción contráctil. El daño miocárdico se acompaña de apoptosis y disfunción ventricular ${ }^{(1,28)}$.

El medio metabólico asociado con la diabetes -hiperglicemia, incremento de ácidos grasos circulantes y triglicéridos, hiperinsulinemia, incremento del estrés oxidativo y de citoquinas inflamatorias y lipotoxicidad- altera múltiples caminos moleculares -vías de señalización-de los cardiomiocitos, entre los cuales se destacan la señalización de la insulina y del sistema renina-angiotensina-aldosterona, la disfunción mitocondrial, la modificación postraduccional de proteínas estructurales y de señalización, la generación de productos finales de glicosilación avanzada, la alteración de los procesos homeostáticos, como apoptosis y autofagia, y estrés del retículo endoplásmico y los cambios en la regulación de genes como activación de factores transcripcionales y mecanismos de micro RNA y epigenéticos. La alteración de las vías moleculares de señalización compromete el manejo del calcio, daña la contractilidad cardíaca y promueve disfunción miocítica, fibrosis intersticial, injuria y muerte celular y produce disfunción miocárdica ${ }^{(27)}$.

En modelos preclínicos de CMD, en ratones, están regulados al alza el factor transcripcional del receptor activado por proliferadores peroxisomales (PPAR) alfa y sus objetivos transcripcionales, lo que lleva al incremento en la betaoxidación y reducción en la utilización de la glucosa, patrón típico de la diabetes. Estos ratones transgénicos PPAR alfa tienen hipertrofia ventricular izquierda, disfunción contráctil y acumulación de lípidos en las células musculares cardíacas. Este fenotipo cardíaco inducido en ratones por sobreexpresión de PPAR alfa imita al fenotipo de la CMD en humanos ${ }^{(30)}$.

Los micro RNA, pequeñas moléculas de RNA que regulan múltiples procesos a través de la transcripción y la expresión postraduccional de genes, participan en la comunicación entre células y están implicados en procesos fisiopatológicos presentes 
en la CMD, entre los cuales se señalan: la regulación de la hipertrofia de los cardomiocitos, la fibrosis miocárdica, la disfunción mitocondrial, la apoptosis, el remodelado eléctrico del miocardio y modificaciones epigenéticas ${ }^{(31)}$. El genoma humano codifica más de 1.000 micro RNA ${ }^{(31)}$. En un modelo animal se comprobó un mecanismo de expresión genética y de hipertrofia de cardiomiocitos mediado a través de la reducción del micro RNA $133^{(32)}$.

Estudios experimentales en modelos animales y avances en técnicas de imagen sugieren que la CMD es una enfermedad progresiva que comienza precozmente luego de la aparición de diabetes tipo 1 y tipo 2 , antes del remodelado del ventrículo izquierdo y de que se manifieste la disfunción diastólica. La activación de la proteína quinasa $\mathrm{C}$-enzima que opera como traductor de señales metabólicas-, parece dirigir vías dependientes y vías no dependientes del estrés oxidativo en el desarrollo de la disfunción contráctil. Se producen mútliples cambios subcelulares: alteración del manejo del calcio, supresión de enzimas productoras de energía aeróbica, modificación transcripcional y postraduccional de proteínas citoesqueléticas de membrana y sarcoméricas, reducción de la dinámica de los puentes de actina miosina y cambios de la sensibilidad al calcio de los miofilamentos, que se destacan actualmente como eventos críticos en las precoces alteraciones en la tasa desarrollo de fuerza, en la relajación y en la estabilidad bajo situaciones de estrés fisiológico o patológico ${ }^{(33)}$.

En la CMD, debido a las alteraciones metabólicas por la hiperglicemia, la hiperinsulinemia y la resistencia a la insulina, ocurre primero disfunción diastólica del ventrículo izquierdo y más tarde disfunción sistólica e insuficiencia cardíaca ${ }^{(28)}$.

La alteración de la perfusión miocárdica puede estar presente con o sin enfermedad aterosclerótica concomitante de las arterias coronarias epicárdicas, por disfunción endotelial que determina disminución de la vasodilatación mediada por flujo y eventualmente también por disminución de la vasodilatación no dependiente de endotelio.

La disfunción endotelial está en la base de la disfunción microvascular que se comprueba en gran parte de la población de pacientes con diabetes. A nivel coronario puede estar presente aun en ausencia de aterosclerosis coronaria y predispone a la misma y a sus complicaciones ${ }^{(34)}$.

La disfunción endotelial periférica, determinada por el estudio de vasodilatación mediada por flujo de la arteria humeral, y la disfunción endotelial coronaria están fuertemente correlacionadas ${ }^{(35)}$.

La dilatación mediada por flujo de la arteria humeral, estudio de ecografía vascular, es el método más confiable para determinar disfunción endotelial peri- férica(36). Aporta buena información pronóstica de acuerdo con el metaanálisis que estimó un $10 \%$ menos de muerte y complicaciones cardiovasculares no fatales por cada $1 \%$ más de dilatación mediada por flu$\mathrm{jo}^{(37)}$.

En una cohorte prospectiva de mujeres posmenopáusicas clínicamente sanas, la presencia de disfunción endotelial periférica se asoció en análisis multivariado al desarrollo futuro de diabetes mellitus tipo $2^{(38)}$.

Se ha comprobado disfunción endotelial periférica en diferentes poblaciones: en familiares directos de personas con diabetes tipo 2 , en intolerantes a la glucosa y en prediabéticos, en obesos y también en diabé$\operatorname{ticos}^{(39)}$. La resistencia a la insulina por sí misma, aun en ausencia de glucosa aumentada, se asocia a disfunción endotelial ${ }^{(39)}$.

La capacidad funcional aeróbica, ajustada para edad y sexo, de personas con síndrome metabólico está disminuida y se asocia a la disfunción endotelial ${ }^{(40)}$. En pacientes con insuficiencia cardíaca por fallo sistólico la capacidad funcional disminuida se asocia también a la disfunción endotelial periférica y se correlaciona con la incidencia de eventos cardiovasculares adversos y mortalidad ${ }^{(41)}$. Puede postularse que la capacidad funcional disminuida de personas con CMD se relaciona con disfunción endotelial periférica y disfunción endotelial coronaria, junto a la disfunción diastólica y sistólica del miocardio.

Se ha demostrado, mediante múltiples estudios transversales y prospectivos, el valor pronóstico de la función endotelial en personas con diabetes como marcador de morbilidad y riesgo cardiovascular ${ }^{(42)}$. La disfunción endotelial es predictor de riesgo de enfermedad cardíaca y renal, lo que sugiere que nuevas intervenciones preventivas y terapéuticas deberían ser recomendadas precozmente para disminuir la morbilidad en esta población de alto riesgo ${ }^{(42)}$.

La identificación de estadios precoces de la enfermedad aterosclerótica en personas con diabetes es un paso fundamental en los protocolos de estratificación de riesgo para tener una visión de conjunto del estadio clínico de un sujeto individual ${ }^{(39)}$. El espesor íntima-media y la detección de placas de ateroma carotídeas subclínicas, la vasodilatación mediada por flujo de la arteria humeral y la velocidad de onda de pulso son herramientas capaces de detectar la precoz alteración del sistema cardiovascular y estratificar el riesgo de los individuos ${ }^{(39)}$.

\section{Manifestaciones y síntomas}

La CMD es una condición frecuente y subdiagnosticada, especialmente en estadios precoces, y puede ser asintomática durante años. 
En la diabetes puede ocurrir precozmente disfunción cardíaca subclínica, que puede estar presente en adolescentes con diabetes tipo $1^{(43)}$ y tipo 2 ${ }^{(44)}$, y se asocia a compromiso de la capacidad de ejercicio o funcional.

La CMD determina por sí misma un riesgo adicional de internaciones por insuficiencia cardíaca, de muerte súbita y de muerte cardiovascular(9).

Los diabéticos sin enfermedad arterial coronaria experimentan excesiva carga de disfunción cardíaca, desde hipertrofia ventricular subclínica a CMD con manifestaciones clínicas, la que es más pronunciada en mujeres ${ }^{45}$.

En pacientes hospitalizados por insuficiencia cardíaca congestiva, con función sistólica ventricular izquierda preservada, la diabetes fue muy prevalente, particularmente en mujeres $(45 \%)$, con frecuentes condiciones comórbidas ${ }^{(46)}$.

Si bien el diagnóstico tardío de diabetes tipo 2 puede explicar el daño de órgano blanco al momento de descubrir la enfermedad, se reconoce que la resistencia a la insulina y sus múltiples consecuencias metabólicas y vasculares pueden determinar disfunción diastólica del ventrículo izquierdo y cardiomiopatía, aun antes del desarrollo de diabetes, lo que puede vincular a la obesidad con la cardiomiopatía en forma independiente de la diabetes ${ }^{(45)}$.

\section{Diagnóstico de cardiomiopatía diabética y sus estadios o fenotipos clínicos}

El diagnóstico de CMD se establece cuando en una persona con diabetes se demuestra disfunción ventricular, sintomática o asintomática, no atribuible a otra enfermedad cardíaca relevante.

En base al resultado de estudios epidemiológicos, clínicos y experimentales, Maish propuso ${ }^{(20)}, \mathrm{y}$ Gilka adaptó(21), en el presente año en base a nuevos conocimientos $^{(47)}$ una clasificación por estadios o fenotipos clínicos de la cardiomiopatía en diabéticos que pueden ser reconocidos mediante estudios serológicos del metabolismo y de marcadores biológicos y por estudios de imagen, asociados a la clínica, que determinan la presencia de alteraciones moleculares, metabólicas, celulares, funcionales y estructurales del miocardio (tabla 1 ).

Si bien la correspondencia entre las diferentes manifestaciones clínicas, las serológicas a través de marcadores biológicos y metabólicos, y las funcionales y estructurales mediante estudios de imagen, seguramente no será exacta para catalogar a una persona en un estadio $u$ otro de CMD, pensamos que este intento de clasificación es una guía útil y está avalada por estudios de seguimiento, ya que se basa en determinaciones que se asocian a significa- tiva predicción independiente del riesgo cardiovascular. La utilización de las herramientas diagnósticas, en aplicación de la propuesta de clasificación de la CMD por estadios, puede mejorar la evaluación del riesgo cardiovascular en personas con diabetes y, al permitir establecer el diagnóstico precoz de CMD, puede precisar aun mejor la intensidad de las medidas correctivas necesarias para optimizar el pronóstico vital y funcional.

\section{Estudios}

La detección precoz puede realizarse mediante estudios serológicos y de imagen que pesquisan la disfunción ventricular aun asintomática.

\section{Biomarcadores}

Más allá de los estudios metabólicos en pacientes con diabetes, varios marcadores de diversos procesos fisiopatológicos pueden ser de utilidad diagnóstica y pronóstica ${ }^{(47)}$.

En un estudio recientemente publicado de dos cohortes prospectivas de diabéticos tipo 2 se demostró el valor predictivo del riesgo de eventos cardiovasculares de tres biomarcadores serológicos: la metaloproteinasa matriz tipo 3 (MMP 3), el fragmento $\mathrm{N}$ terminal pro-hormona del péptido natriurético tipo cerebral (NT-proBNP) y la proteína osteopontina, que representan diferentes vías fisiopatológicas y mejoraron la predicción del riesgo de eventos cardiovasculares más allá de los factores de riesgo tradicionales ${ }^{(47)}$.

El NT-proBNP, un polipéptido secretado por los cardiomiocitos en respuesta al estiramiento y a la tensión de la pared, mostró el mayor potencial predictivo individual en dicho estudio ${ }^{(47)}$. El NT-proBNP en pacientes asintomáticos con diabetes 2 pudo excluir precozmente la disfunción del ventrículo izquierdo determinada por eco Doppler pulsado, con alto valor predictivo negativo $(99,5 \%)$, utilizando el punto de corte de $125 \mathrm{pg} / \mathrm{ml}^{(48)}$. Valores mayores sugirieron la necesidad de realizar un estudio de imagen para confirmar la presencia de disfunción ventricular asintomática.

La MMP-3 es una enzima que puede degradar el tejido conectivo de la matriz de la cual depende la estabilidad de la placa aterosclerótica.

La osteopontina es una glicoproteína ligada al calcio que ha sido implicada en la inmunidad celular y en la progresión de la aterosclerosis y se asocia en forma independiente al riesgo cardiovascular ${ }^{(47)}$.

Estos tres biomarcadores aportaron valor predictivo en forma independiente y también complementaria, de manera que el abordaje de múltiples biomarcadores tuvo el mejor desempeño(47). 
Tabla 1. Clasificación de estadios o fenotipos clínicos de cardiomiopatía diabética, adaptada de Maish ${ }^{(20)}$ y Gilka ${ }^{(21)}$.

\begin{tabular}{|c|c|c|c|c|}
\hline & $\begin{array}{l}\text { Estadio } 1 \\
\text { Disfunción diastólica, } \\
\text { bipertrofia }\end{array}$ & $\begin{array}{l}\text { Estadio } 2 \\
\text { Disfunción sistólicay } \\
\text { dilatación }\end{array}$ & $\begin{array}{l}\text { Estadio } 3 \\
\text { Disfunción sistólica y } \\
\text { dilatación, } H A \text { asociada }\end{array}$ & $\begin{array}{l}\text { Estadio } 4 \\
\text { Incluyendo todos los factores } \\
\text { de confusión, incluso EAC }\end{array}$ \\
\hline $\begin{array}{l}\text { Clasificación de la } \\
\text { NYHA }\end{array}$ & Asintomático & Clase II & Clase II-III & Clase II-IV \\
\hline Metabolismo & $\begin{array}{l}\text { Intolerancia a la } \\
\text { glucosa, síndrome } \\
\text { metabólico }\end{array}$ & Hiperglicemia crónica & $\begin{array}{l}\text { Resistencia a la } \\
\text { insulina, diabetes con } \\
\text { complicaciones } \\
\text { microangiopáticas }\end{array}$ & $\begin{array}{l}\text { Con complicaciones } \\
\text { micro y } \\
\text { macroangiopáticas }\end{array}$ \\
\hline $\begin{array}{l}\text { Ecocardiografía +/- } \\
\text { CACG }\end{array}$ & $\begin{array}{l}\text { Disfunción diastólica } \\
\text { con FEVI normal, } \\
\text { disminución de las } \\
\text { velocidades diastólicas } \\
\text { tisulares en el Doppler } \\
\text { tisular, con aumento } \\
\text { de la masa del VI, sin } \\
\text { HA, EAC ni } \\
\text { valvulopatía relevante }\end{array}$ & $\begin{array}{l}\text { Disfunción diastólica } \\
\text { y sistólica con } \\
\text { dilatación y FEVI } \\
\text { disminuida, } \\
\text { excluyendo relevante } \\
\text { EAC, valvulopatía o } \\
\text { HA no controlada }\end{array}$ & $\begin{array}{l}\text { Disfunción sistólica y } \\
\text { diastólica, con } \\
\text { enfermedad } \\
\text { microvascular y/o } \\
\text { infección microbiana } \\
\text { y/o inflamación y/o } \\
\text { HA, sin EAC }\end{array}$ & $\begin{array}{l}\text { CACG si el fallo } \\
\text { cardíaco puede ser } \\
\text { atribuido a infarto o } \\
\text { isquemia y } \\
\text { remodelado, además } \\
\text { de a estadio } 3 \text { de CMD }\end{array}$ \\
\hline $\begin{array}{l}\text { Otras } \\
\text { comorbilidades } \\
\text { asociadas a diabetes }\end{array}$ & & & $\begin{array}{l}\text { Complicaciones } \\
\text { microangiopáticas. } \\
\text { HA. }\end{array}$ & $\begin{array}{l}\text { Complicaciones } \\
\text { macroangiopáticas, } \\
\text { incluyendo EAC. }\end{array}$ \\
\hline $\begin{array}{l}\text { Marcadores } \\
\text { serológicos a } \\
\text { monitorear } \\
\text { periódicamente para } \\
\text { control metabólico, } \\
\text { de fallo cardíaco y } \\
\text { necrosis }\end{array}$ & $\begin{array}{l}\text { NT-proBNP, MMP3 y } \\
\text { Osteopontina (de } \\
\text { acuerdo a Van Der } \\
\text { Leew, et al. 2016) }{ }^{(47)} \text {. } \\
\text { Glicemia, HbA1c y } \\
\text { lipidograma }\end{array}$ & $\begin{array}{l}\text { MMP3, Osteopontina } \\
\text { (de acuerdo a Van Der }^{(47)} \\
\text { Leew, et al. 2016) } \\
\text { Glicemia, HbA1c y } \\
\text { lipidograma, } \\
\text { NT-proBNP y } \\
\text { ProBNP. }\end{array}$ & $\begin{array}{l}\text { NT-proBNP, MMP3 y } \\
\text { Osteopontina. } \\
\text { Glicemia, HbA1c y } \\
\text { lipidograma, } \\
\text { NT-proBNP y } \\
\text { ProBNP. } \\
\text { La troponina aumenta } \\
\text { si hay isquemia }\end{array}$ & $\begin{array}{l}\text { NT-proBNP, MMP3 y } \\
\text { Osteopontina. } \\
\text { Glicemia, HbA1c y } \\
\text { lipidograma, ProBNP. } \\
\text { Troponina aumentada } \\
\text { si hay infarto } \\
\text { miocárdico o fallo } \\
\text { cardíaco severo }\end{array}$ \\
\hline
\end{tabular}

\section{Electrocardiograma}

En un estudio de personas con diabetes tipo 2 y sin isquemia en el estudio funcional, la escasa progresión de las ondas $\mathrm{R}$ del electrocardiograma en las derivaciones precordiales V1 a V3, definida como ondas $\mathrm{R}$ menores de $3 \mathrm{~mm}$, se asoció a peor función diastólica del ventrículo izquierdo evaluada por el índice de desempeño miocárdico de Tei con el Doppler pulsado y por la velocidad del anillo mitral con el Doppler tisular y se asoció, en el seguimiento a cuatro años, a mayor disfunción sistólica con disminución de la fracción de eyección del ventrículo izquierdo y a empeoramiento de la función diastólica(49). De esta forma, la escasa progresión de la onda $R$ del electrocardiograma puede alertar sobre la posibilidad de CMD.

\section{Técnicas de imagen}

En el diagnóstico de CMD varios métodos de imagen pueden detectar precozmente disfunción ventricular.

\section{Ecocardiografía}

De los estudios de imagen se destaca el ecocardiograma por su información y disponibilidad. Permite alcanzar el diagnóstico de CMD incluso antes de los síntomas. El hallazgo precoz más frecuente en diabéticos asintomáticos es la disfunción diastólica del ventrículo izquierdo sin hipertrofia asociada. El Doppler tisular es más sensible para detectar disfunción diastólica. Mediante speckle-tracking puede detectarse en forma precoz, aun en diabéticos asintomáticos no complicados, disfunción sistólica evidenciada por disminución del strain longitudinal del ventrículo izquierdo ${ }^{(50,51)}$.

La diabetes por sí misma, en ausencia de hipertensión arterial y de enfermedad arterial coronaria, es una determinante independiente, en análisis multivariado, del fenotipo y la función del ventrículo izquierdo, especialmente del aumento de su masa, de remodelado concéntrico, del compromiso de su relajación, de la fracción de acortamiento, de la 
función sistólica longitudinal y de la fracción de eyección, lo que puede objetivarse incluso en pacientes asintomáticos mediante ecocardiograma convencional, tisular y speckle tracking ${ }^{(51)}$.

La concomitancia de hipertensión arterial acentúa la disfunción diastólica y la disfunción sistólica. La enfermedad arterial coronaria concomitante compromete aún más la fracción de acortamiento y la excursión del plano del anillo mitral. El strain circunferencial pico se altera solo en caso de que la diabetes se asocie a hipertensión arterial o a enfermedad arterial coronaria ${ }^{(51)}$.

\section{Resonancia magnética cardíaca}

De los métodos que pueden emplearse cuando la calidad de las imágenes del ecocardiograma no es buena o por su aporte intrínseco, se destaca la resonancia magnética cardíaca, que puede detectar la hipertrofia y la esteatosis miocárdica, así como la disfunción diastólica y sistólica del ventrículo izquier$\mathrm{do}^{(52)}$. En la resonancia magnética cardíaca el realce tardío con gadolinium detectó fibrosis intersticial en $28 \%$ de los pacientes con diabetes sin evidencia clínica de infarto miocárdico ${ }^{(52)}$.

\section{Otros estudios}

La tomografía con emisión de positrones, poco disponible por sus costos, puede dar información metabólica sobre el miocardio ${ }^{(51)}$.

Los estudios funcionales con estrés -ejercicio o fármacos- para detectar isquemia miocárdica o la angiografía coronaria se indican si se sospecha enfermedad arterial coronaria, eligiendo el tipo de estudio según las características del cuadro clínico.

\section{Tratamiento}

\section{Hábitos saludables}

Los hábitos saludables de vida que incluyen ejercicio, nutrición adecuada y peso saludable previenen y mejoran la resistencia a la insulina y la diabetes y así disminuyen la incidencia de CMD y de muerte prematura.

El ejercicio físico reduce significativamente el riesgo de eventos cardiovasculares. Induce cardioprotección por modulación de los factores de riesgo sistémicos y de la función vascular y endotelial y directamente sobre el desempeño cardíaco, disminuyendo la incidencia de disfunción cardíaca. El ejercicio de volumen e intensidad adecuados puede mejorar la disfunción miocárdica a través de mejoría del consumo máximo de oxígeno, de la fracción de eyección y de los volúmenes diastólico y sistólico del ven- trículo izquierdo, del gasto cardíaco y de la disfunción diastólica ${ }^{(53)}$.

Se ha propuesto un diálogo cruzado entre el músculo esquelético ejercitado y el músculo cardíaco y las paredes arteriales a través de comunicación por micro RNA, posibles llaves reguladoras de múltiples procesos fisiopatológicos ${ }^{(53)}$.

\section{Tratamiento multifactorial}

El tratamiento debe dirigirse a lograr una nutrición con menor índice glicémico, optimizar la actividad física, el peso corporal, la grasa visceral, la resistencia a la insulina, la función endotelial, la presión arterial y los metabolismos glucídico y lipídico ${ }^{(55,56)}$.

En el trabajo Steno Study de diabéticos tipo 2 con microalbuminuria ${ }^{(55,56)}$, el tratamiento multifactorial intensivo de los factores de riesgo cardiovascular y metabólico con hábitos saludables y fármacos, comparado con el tratamiento convencional, demostró gran poder para prevenir a mediano y largo plazo el desarrollo de complicaciones cardiovasculares y microvasculares, muerte total y muerte cardiovascular. El grupo de tratamiento intensivo multifactorial randomizado durante los primeros ocho años, en el seguimiento promedio de 21 años, logró ocho años de vida ganados al compararlo con el tratamiento convencional ${ }^{(57)}$. El incremento de la esperanza de vida fue igualado por el tiempo libre de enfermedad cardiovascular incidente. La muerte por causas cardiovasculares se redujo $62 \%$ en el grupo de tratamiento intensivo, sin diferencia en la mortalidad no cardiovascular entre los grupos.

No hubo diferencias significativas entre los grupos en la distribución del tipo específico de primer evento cardiovascular ${ }^{(57)}$.

Basándose en esos beneficios se debe implementar un plan de tratamiento integral e intensivo multifactorial de los factores de riesgo metabólico y cardiovascular ${ }^{(54)}$ para la prevención de las lesiones de los "órganos blanco"(55,56), dirigido al control de la presión arterial, los lípidos y la hemoglobina glicosilada (HbA1c) ${ }^{(24,58)}$.

El tratamiento concomitante de los múltiples factores de riesgo produjo mayores reducciones del riesgo que el tratamiento de un factor. En el estudio VADT, alcanzar los dos objetivos de HbA1c y de LDL colesterol determinó mayores reducciones del riesgo que alcanzar uno solo de ellos ${ }^{(59)}$, y en el estudio randomizado $\mathrm{ADVANCE}$ de control intensivo de la glucosa con gliclazida y de reducción intensiva de la presión arterial, al agregar al tratamiento perindopril/indapamida en un comprimido, la mortalidad total fue menor(60). 


\section{Tratamiento antidiabético}

El tratamiento farmacológico de la resistencia a la insulina y de control glucémico debe ser conducido por un especialista endocrinólogo o diabetólogo. El tratamiento de control glicémico puede ser también beneficioso por reducción de la esteatosis hepática ${ }^{(61)}$. La disminución de la incidencia de la CMD en diabetes tipo 2 por el control intensivo de la glicemia no se ha demostrado todavía(50).

La metformina mejora la resistencia a la insulina y aumenta la autofagia, proceso intracelular que desempeña un rol en la prevención de la CMD. El estudio UKPDS demostró disminución de los eventos cardiovasculares con metformina ${ }^{(62)}$, motivo por el cual es el tratamiento de primera línea en diabetes tipo $2^{(63)}$. En pacientes con diabetes, sobrepeso e insuficiencia cardíaca, a pesar del infrecuente riesgo de acidosis láctica, la metformina reduce la mortalidad y mejora la evolución clínica ${ }^{(51,64)}$.

Péptidos similares al glucagón (GLP-1) atenúan la apoptosis miocárdica y aumentan la vasodilatación $8^{(51)}$

Se ha comunicado que inhibidores DPP-4 o gliptinas pueden prevenir la disfunción diastólica por inhibición de la fibrosis y del estrés oxidativo ${ }^{(51)}$. No se han encontrado diferencias relevantes en el riesgo de eventos cardiovasculares, incluyendo las hospitalizaciones por insuficiencia cardíaca, entre tres de las gliptinas y el placebo ${ }^{(65)}$.

La empagliflozina, un potente y selectivo inhibidor del cotransportador 2 de sodio-glucosa (SGLT2) en diabetes tipo 2, reduce la hiperglicemia, la hiperinsulinemia, el estrés oxidativo, el peso corporal, la presión arterial y los marcadores de resistencia vascular y de rigidez arterial ${ }^{(66)}$. En el estudio EMPA-REG OUTCOME la empagliflozina agregada al tratamiento estándar disminuyó las hospitalizaciones por insuficiencia cardíaca y la mortalidad total y cardiovascular, comparado con placebo, en pacientes con diabetes 2 y alto riesgo cardiovascular ${ }^{(67)}$, con o sin fallo cardíaco previo ${ }^{(68)}$.

\section{Estatinas}

Las estatinas reducen la fibrosis miocárdica y la inflamación y mejoran la función del ventrículo izquierdo, disminuyen la presión arterial al mejorar las propiedades arteriales, además de disminuir en diabéticos los eventos cardiovasculares y la muerte cardiovascular y total ${ }^{(51,63)}$. Por cada $40 \mathrm{mg}$ (1 mMol) de disminución del LDL colesterol con el tratamiento de diabéticos con estatinas se reduce $21 \%$ el riesgo de complicaciones cardiovasculares ${ }^{(69)}$.

En personas con diabetes 2, aun con nivel de colesterol LDL basal menor de $130 \mathrm{mg} / \mathrm{dl}$, la atorvastatina en dosis de $10 \mathrm{mg}$ al día en el estudio CARDS demos- tró beneficios con una reducción del 37\% de los eventos cardiovasculares ${ }^{(70)}$. Con altas dosis de estatinas se logra una mayor disminución de las complicaciones cardiovasculares de diabéticos en prevención secundaria de aterosclerosis y en otros diabéticos con alto riesgo cardiovascular, de acuerdo al estudio NNT que demostró $25 \%$ de reducción de eventos cardiovasculares con la dosis diaria de $80 \mathrm{mg}$ de atorvastatina, comparada con la dosis de $10 \mathrm{mg}$ de la misma droga ${ }^{(71)}$.

\section{Medicamentos vasoactivos}

Los inhibidores de la enzima conversora de la angiotensina (IECA) y los antagonistas de receptores AT II (ARA II) revierten los índices ecográficos precoces de CMD ${ }^{(72)}$. En el subestudio MICRO-HOPE de diabéti$\cos 2$ con alto riesgo cardiovascular-con promedio de presión arterial basal en rango de presión arterial normal-alta-, ramipril redujo los eventos cardiovasculares $25 \%$ y la mortalidad total $24 \%{ }^{(73)}$. Los IECA y los ARA II mejoran la insuficiencia cardíaca y reducen su mortalidad ${ }^{(51,63)}$.

De las combinaciones fijas de dos antihipertensivos, la de IECA y bloqueante de canal cálcico, y la de ARA II y bloqueante de canal cálcico, produjeron mayores regresiones de la disfunción diastólica aislada del ventrículo izquierdo en hipertensos, que las otras asociaciones de IECA con diurético, ARA II con diurético y betabloqueante con diurético, con similar reducción de la presión arterial ${ }^{(74)}$. En el mismo estudio, en los hipertensos que asociaban glicemia de ayuno alterada, la combinación de IECA y bloqueante de canal cálcico produjo mayor regresión de la disfunción diastólica aislada del ventrículo izquierdo, que la de ARA II y bloqueante de canal cálcico(74). Se recomiendan los IECA como antihipertensivos de primera línea en pacientes con diabetes.

Los betabloqueantes disminuyen las hospitalizaciones y la mortalidad y mejoran los síntomas de insuficiencia cardíaca ${ }^{(51)}$

El sildenafil, un inhibidor de la fosfodiesterasa, mejora la función y el remodelado cardíacos, así como algunos marcadores de inflamación cardíaca en pacientes con $\mathrm{CMD}^{(51,75)}$. En un estudio in vitro de cultivos de células humanas mononucleares de la sangre, sildenafil reguló a la baja la producción y la expresión genética de la osteopontina ${ }^{(76)}$. Resta estudiar el efecto de sildenafil en pacientes que tienen niveles aumentados de osteopontina, como los que son portadores de diabetes tipo 2, o de enfermedades cardiovasculares, hepáticas o autoinmunes ${ }^{(76)}$.

El tratamiento farmacológico integral de prevención cardiovascular debe incluir estatinas y fármacos para lograr los objetivos de control óptimo de la diabetes y de la presión arterial. 


\section{Moduladores metabólicos}

Los resultados de estudios con algunas moléculas con efecto metabólico permiten plantearlas como promisorias en esta situación.

La trimetazidina reduce el daño por radicales libres, mejora la función endotelial, inhibe la apoptosis y atenúa la lipotoxicidad, por lo que promete tener efectos beneficiosos en pacientes con diabetes y fallo cardíaco por cardiomiopatía dilatada isquémica y también por $\mathrm{CMD}^{(51,77)}$. Los resultados de un metaanálisis señalan que la trimetazidina disminuye en $53 \%$ las hospitalizaciones por insuficiencia cardía $\mathrm{ca}^{(77)}$.

El ácido alfa lipoico, potente antioxidante natural, tiene efectos beneficiosos en modelos experimentales en la homeostasis redox y en la supresión de la fibrosis cardíaca $^{(51,78)}$.

En un estudio de pacientes con diabetes tipo 2, el antioxidante específico ácido alfa lipoico administrado por vía intravenosa mejoró la vasodilatación dependiente de endotelio ${ }^{(79)}$.

La coenzima Q10, antioxidante lipídico intermediario en el transporte de electrones y la fosforilación oxidativa de la cadena respiratoria, mejora la función cardíaca en insuficiencia cardíaca concomitante con diabetes ${ }^{(80)}$. Un estudio randomizado mostró que el tratamiento a largo plazo con la coenzima Q10 en pacientes con insuficiencia cardíaca crónica es seguro, mejora los síntomas y reduce la mortalidad por todas las causas en $42 \%$ y la mortalidad cardiovascular en $43 \%{ }^{(81)}$.

El resveratrol es un antioxidante natural sintetizado en numerosas plantas como respuesta a infecciones o radiación ultravioleta. A diferencia de otros metabolitos de origen natural, niveles de resveratrol clínicamente relevantes son difíciles de obtener por la dieta. Está siendo ampliamente estudiado por su participación en numerosas vías de señalización vinculadas al desarrollo de inflamación y de enfermedades crónicas cardiovasculares, neurológicas y cáncer, y por su potencial preventivo y terapéutico.

De los estudios en animales se destaca que mejoró la salud y prolongó la vida de ratones alimentados con dieta hipercalórica. El análisis del enriquecimiento de conjunto de genes reveló que el resveratrol se opuso al efecto de la dieta hipercalórica en 144 de 153 vías de señalización significativamente alteradas ${ }^{(82)}$, simulando el efecto de la restricción calórica ${ }^{(83)}$. En ratones con insuficiencia cardíaca por CMD, el resveratrol redujo la glucosa en sangre y mejoró la sensibilidad a la insulina y la función cardíaca ${ }^{(84)}$.

En estudios en humanos ha demostrado mejoría de la función endotelial en adultos obesos ${ }^{(85)}$ y en un grupo de pacientes que se recuperaban de infarto de miocardio( ${ }^{(86)}$, así como mejoría de la capacidad mito- condrial del músculo esquelético ${ }^{(87)}$. En un estudio controlado con placebo de mujeres posmenopáusicas tratadas durante 14 semanas, el grupo tratado con resveratrol tuvo mejor desempeño cognitivo, humor y memoria, que al menos en parte se correlacionó con el aumento del flujo sanguíneo cerebral( ${ }^{(88)}$.

De los ensayos clínicos randomizados se destacan en pacientes con diabetes tipo 2 la mejoría de la sensibilidad a la insulina y la disminución del estrés oxidativo ${ }^{(89)}$; la disminución del tamaño de las úlceras de los pies $^{(90)}$, y el metaanálisis de 196 pacientes que mostró que los tratados con resveratrol tuvieron menores niveles de presión arterial sistólica, hemoglobina glicosilada y creatinina, con buen perfil de seguridad, postulándolo como posible adyuvante al tratamiento farmacológico en la diabetes ${ }^{(91)}$.

\section{Conclusiones}

La CMD se desarrolla por las alteraciones metabólicas que desencadenan disfunción y daño miocárdico, y que se asocian a exceso de mortalidad.

El diagnóstico de CMD se establece por la detección de disfunción ventricular no atribuible a otra enfermedad cardíaca relevante.

La prevención, el diagnóstico precoz y el tratamiento adecuado de la CMD pueden mejorar la calidad de vida y el pronóstico vital.

\section{Bibliografía}

1. Wang ZV, Hill JA. Diabetic cardiomyopathy: catabolism driving metabolism. Circulation 2015; 131(9):771-3. doi: 10.1161/CIRCULATIONAHA.115.015357.

2. NCD Risk Factor Collaboration (NCD-RisC). Worldwide trends in diabetes since 1980: a pooled analysis of 751 population-based studies with $44 \mathrm{mi}-$ llion participants. Lancet 2016; 387(10027):1513-30. doi: 10.1016/S0140-6736(16)00618-8.

3. Rubinstein A, Irazola V, Calandrelli M, Elorriaga N, Gutierrez L, Lanas F, et al. Multiple cardiometabolic risk factors in the Southern Cone of Latin America: A population-based study in Argentina, Chile, and Uruguay. Int J Cardiol 2015; 183:82-8. doi: 10.1016/j.ijcard.2015.01.062.

4. Kannel WB, Hjortland M, Castelli WP. Role of diabetes in congestive heart failure: the Framingham study. Am J Cardiol 1974; 34(1):29-34.

5. Mosterd A, Hoes AW. Clinical epidemiology of heart failure. Heart 2007; 93(9):1137-46. doi: 10.1136/hrt.2003.025270.

6. Sakata Y, Shimokawa H. Epidemiology of heart failure in Asia. Circ J 2013; 77(9):2209-17. 
7 Bocchi EA, Braga FG, Ferreira SM, Rhode LE, Oliveira WA, Almeida DR, et al. III Diretriz Brasileira de Insuficiência Cardíaca Crônica. Arq Bras Cardiol 2009; 93(1 Supl 1):3-70.

8. Ambrosy AP, Fonarow GC, Butler J, Chioncel O, Greene SJ, Vaduganathan M, et al. The global health and economic burden of hospitalizations for heart failure: lessons learned from Hospitalized Heart Failure registries. J Am Coll Cardiol 2014; 63(12):1123-33. doi: 10.1016/j.jacc.2013.11.053.

9. Drozd M, Kearney MT. Diabetes mellitus and heart failure: a deadly duo. J Thorac Dis 2017; 9(1):16-8. doi: 10.21037/jtd.2017.01.24.

10. Cubbon RM, Adams B, Rajwani A, Mercer BN, Patel PA, Gherardi G, et al. Diabetes mellitus is associated with adverse prognosis in chronic heart failure of ischaemic and non-ischaemic aetiology. Diab Vasc Dis Res 2013; 10(4):330-6. doi: 10.1177/1479164112471064.

11. Nichols GA, Hillier TA, Erbey JR, Brown JB. Congestive heart failure in type 2 diabetes: prevalence, incidence, and risk factors. Diabetes Care 2001; 24(9):1614-9

12. Boudina S, Abel ED. Diabetic cardiomyopathy revisited. Circulation. 2007; 115(25):3213-23

13. Dries DL, Sweitzer NK, Drazner MH, Stevenson LW, Gersh BJ. Prognostic impact of diabetes mellitus in patients with heart failure according to the etiology of left ventricular systolic dysfunction. $J$ Am Coll Cardiol 2001; 38(2):421-8.

14. Aneja A, Wilson Tang WH, Bansilal S, García MJ, Farkouh ME. Diabetic cardiomyopathy: insights into pathogenesis, diagnostic challenges, and therapeutic options. Am J Med 2008; 121(9):748-57. doi: 10.1016/j.amjmed.2008.03.046.

15. Rubler S, Dlugash J, Yuceoglu YZ, Kumral T, Branwood AW, Grishman A. New type of cardiomyopathy associated with diabetic glomerulosclerosis. Am J Cardiol. 1972; 30(6):595-602.

16. Bertoni AG, Hundley WG, Massing MW, Bonds DE, Burke GL, Goff DC Jr. Heart failure prevalence, incidence, and mortality in the elderly with diabetes. Diabetes Care 2004; 27(3):699-703.

17. Wong AKF, AlZadjali MA, Choy AMJ, Lang CC. Insulin resistance: a potential new target for therapy in patients with heart failure. Cardiovasc Ther 2008; 26(3):203-13. doi: 10.1111/j.1755-5922.2008.00053.x.

18. Doehner W, Rauchhaus M, Ponikowski P, Godsland IF, von Haehling S, Okonko DO, et al. Impaired insulin sensitivity as an independent risk factor for mortality in patients with stable chronic heart failure. J Am Coll Cardiol 2005; 46(6):1019-26.

19. Marwick TH. Diabetic heart disease. Heart. 2006; 92(3):296-300. doi: 10.1136/hrt.2005.067231.
20. Maisch B, Alter P, Pankuweit S. Diabetic cardiomyopathy_fact or fiction?. Herz 2011; 36(2):10215 .

21. Gilca GE, Stefanescu G, Badulescu O, Tanase DM, Bararu I, Ciocoiu M. Diabetic cardiomyophaty: Current approach and potential diagnostic and therapeutic targets. J Diabetes Res 2017; 2017:7p. doi: $10.1155 / 2017 / 1310265$.

22. Boudina S, Abel ED. Diabetic cardiomyopathy, causes and effects. Rev Endocr Metab Disord 2010; 11(1):31-9. doi: 10.1007/s11154-010-9131-7.

23. Westermeier F, Riquelme JA, Pavez M, Garrido V, Díaz A., Verdejo HE, et al. New molecular insights of insulin in diabetic cardiomyopathy. Front Physiol 2016; 7:125. doi: 10.3389/fphys.2016.00125.

24. Wang CCL, Reusch JEB. Diabetes and cardiovascular disease: Changing the focus from glycemic control to improving long-term survival. Am J Cardiol 2012; 110(9 Suppl):58B-68B. doi: 10.1016/j.amjcard.2012.08.036.

25. Harmancey $\mathbf{R}$, Taegtmeyer $\mathbf{H}$. The complexity of diabetic cardiomyopathy: lessons from patients and animal models. Curr Diab Rep 2008; 8(3):243-8. PMC3637972.

26. Burgmaier M, Sen S, Philip F, Wilson CR, Miller CC 3rd, Young ME, et al. Metabolic adaptation follows contractile dysfunction in the heart of obese Zucker rats fed a high-fat "Western" diet. Obesity (Silver Spring) 2010; 18(10):1895-901. doi: 10.1038/oby.2009.500.

27. Bugger H, Abel ED. Molecular mechanisms of diabetic cardiomyopathy. Diabetologia 2014; 57(4):66071.

28. Jia G, De Marco VG, Sowers JR. Insulin resistance and hyperinsulinaemia in diabetic cardiomyopathy. Nat Rev Endocrinol 2016; 12(3):144-53.

29. Wold LE, Ceylan-Isik AF, Ren J. Oxidative stress and stress signaling: menace of diabetic cardiomyopathy. Acta Pharmacologica Sinica 2005; 26:908-17. doi: 10.1111/j.1745-7254.2005.00146.x.

30. Finck BN, Lehman JJ, Leone TC, Welch MJ, Bennett MJ, Kovacs A, et al. The cardiac phenotype induced by PPAR alpha overexpression mimics that caused by diabetes mellitus. J Clin Invest 2002; 109(1):121-30. doi: 10.1172/JCI14080.

31. Liu X, Liu S. Role of microRNAs in the pathogenesis of diabetic cardiomyopathy. Biomed Re. 2017; 6(2):140-5. PubMed PMID: 28357065

32. Rawal S, Ram TP, Coffey S, Williams MJA, Saxena $\mathbf{P}$, Bunton RW, et al. Differential expression pattern of cardiovascular microRNAs in the human type-2 diabetic heart with normal ejection fraction. Int J Cardiol. 2016; 202:40-3. doi: 10.1016/j.ijcard.2015.08.161. 
33. Waddingham MT, Edgley AJ, Tsuchimochi H, Kelly DJ, Shirai M, Pearson JT. Contractile apparatus dysfunction early in the pathophysiology of diabetic cardiomyopathy. World J Diabetes 2015; 6(7):943-60. doi:10.4239/wjd.v6.i7.943.

34. Schächinger V, Britten MB, Zeiher AM. Prognostic impact of coronary vasodilator dysfunction on adverse long-term outcome of coronary heart disease. Circulation 2000; 101(16):1899-906.

35. Anderson TJ, Uehata A, Gerhard MD, Meredith IT, Knab S, Delagrange D, et al. Close relation of endothelial function in the human coronary and peripheral circulations. J Am Coll Cardiol 1995; 26(5):1235-41. Disponible en: http://www.sciencedirect.com/science/article/pii/073510979500327.

36. Onkelinx S, Cornelissen V, Goetschalckx K, Thomaes T, Verhamme P, Vanhees L. Reproducibility of different methods to measure the endothelial function. Vasc Med 2012; 17(2):79-84. doi: $10.1177 / 1358863 \times 12436708$.

37. Xu Y, Arora RC, Hiebert BM, Lerner B, Szwajcer A, McDonald K, et al. Non-invasive endothelial function testing and the risk of adverse outcomes: a systematic review and meta-analysis. Eur Heart J-Cardiovascular Imaging 2014;15(7):736-46. doi: 10.1093/ehjci/jet256.

38. Rossi R, Cioni E, Nuzzo A, Origliani G, Modena MG. Endothelial-Dependent vasodilatation and incidence of type 2 diabetes in a population of healthy postmenopausal women. Diabetes Care 2005; 28(3):702-7. doi: 10.2337/diacare.28.3.702.

39. Ciccone MM, Scicchitano P, Cameli M, Cecere A, Cortese F, Dentamaro I, et al. Endothelia function in pre-diabetes, diabetes and diabetic cardiomyopathy: A review. J Diabetes Metab 2014; 5 : 364. doi: 10.4172/2155-6156.1000364.

40. Manfredi JA, Bermúdez C, Pisabarro R, Gutiérrez $M$. Aerobic capacity and endothelial function in patients with metabolic syndrome [Abstract]. Eur Heart J 2011; 32 Suppl 1:712. doi: 10.1093/eurheartj/ehr324

41. Katz SD, Hryniewicz K, Hriljac I, Balidemaj K, Dimayuga C, Hudaihed A, et al. Vascular endothelial dysfunction and mortality risk in patients with chronic heart failure. Circulation 2005; 111:310-4. doi: 10.1161/01.CIR.0000153349.77489.CF.

42. Ladeia AM, Sampaio RR, Hita MC, Adan LF. Prognostic value of endothelial dysfunction in type 1 diabetes mellitus. World J Diabetes. 2014; 5(5): 601-5. doi: 10.4239/wjd.v5.i5.601.

43. Nadeau KJ, Regensteiner JG, Bauer TA, Brown MS, Dorosz JL, Hull A, et al. Insulin resistance in adolescents with type 1 diabetes and its relationship to cardiovascular function. J Clin Endocrinol Metab. 2010; 95(2):513-21. doi: $10.1210 /$ jc.2009-1756.
44. Nadeau KJ, Zeitler PS, Bauer TA, Brown MS, Dorosz JL, Draznin B, et al. Insulin resistance in adolescents with type 2 diabetes is associated with impaired exercise capacity. J Clin Endocrinol Metab 2009; 94(10):3687-95. doi: 10.1210/jc.2008-2844.

45. Rutter MK, Parise H, Benjamin EJ, Levy D, Larson MG, Meigs JB, et al. Impact of glucose intolerance and insulin resistance on cardiac structure and function: sex-related differences in the Framingham Heart Study. Circulation 2003; 107(3):448-54.

46. Klapholz M, Maurer M, Lowe AM, Messineo F, Meisner JS, Mitchell J, et al; New York Heart Failure Consortium. Hospitalization for heart failure in the presence of a normal left ventricular ejection fraction: results of the New York Heart Failure Registry. J Am Coll Cardiol 2004; 43(8):1432-8. doi: 10.1016/j.jacc.2003.11.040.

47. van der Leeuw J, Beulens JW, van Dieren S, Schalkwijk CG, Glatz JF, Hofker MH, et al. Novel biomarkers to improve the prediction of cardiovascular event risk in type 2 diabetes mellitus. J Am Heart Assoc 2016; 5(6):e003048. doi: 10.1161/JAHA.115.003048

48. Betti I, Castelli G, Barchielli A, Beligni C, Boscherini V, De Luca $L$, et al. The role of N-terminal PRO-brain natriuretic peptide and echocardiography for screening asymptomatic left ventricular dysfunction in a population at high risk for heart failure. The PROBE-HF study. J Card Fail 2009; 15(5):377-84. doi: 10.1016/j.cardfail.2008.12.002.

49. Bildirici U, Ural D, Acar E, Agacdiken A, Ural E. Diagnostic value of poor R-wave progression in electrocardiograms for diabetic cardiomyopathy in type 2 diabetic patients. Clin Cardiol. 2010; 33(9):559-64. doi: 10.1002/clc.20810.

50. Loncarevic B, Trifunovic D, Soldatovic I, Vujisic-Tesic B. Silent diabetic cardiomyopathy in everyday practice: a clinical and echocardiographic study. BMC Cardiovascular Disorders. 2016; 16:242. doi: 10.1186/s12872-016-0395-z.

51. Lee WS, Kim J. Diabetic cardiomyopathy: where we are and where we are going. Korean J Intern Med. 2017; 32(3): 404-21. doi: 10.3904/kjim.2016.208.

52. Shah RV, Abbasi SA, Kwong RY. Role of cardiac MRI in diabetes. Curr Cardiol Rep 2014; 16(2): 449. doi: 10.1007/s11886-013-0449-0.

53. Lew JKS, Pearson JT, Schwenke DO, Katare R. Exercise mediated protection of diabetic heart through modulation of microRNA mediated molecular pathways. Cardiovasc Diabetol. 2017; 16:10. doi: 10.1186/s12933-016-0484-4.

54. Buse JB, Ginsberg HN, Bakris GL, Clark NG, Costa F, Eckel R, et al. Primary prevention of cardiovascular diseases in people with diabetes mellitus: a scientific statement from the American Heart Association and the American Diabetes Association. Cir- 
culation. 2007 ; 115(1): 114-26.

doi: 10.1161/CIRCULATIONAHA.106.179294.

55. Gæde P, Vedel P, Larsen N, Jensen GVH, Parving H-H, Pedersen O. Multifactorial intervention and cardiovascular disease in patients with type 2 diabetes. N Engl J Med. 2003; 348(5): 383-93. doi: 10.1056/NEJMoa021778.

56. Gæde P, Lund-Andersen H, Parving H-H, Pedersen $\mathbf{O}$. Effect of a multifactorial intervention on mortality in type 2 diabetes. N Engl J Med 2008; 358(6):580-91. doi: 10.1056/NEJMoa0706245.

57. Gaede $\mathbf{P}$, Oellgaard J, Carstensen $\mathbf{B}$, Rossing $\mathbf{P}$, Lund-Andersen H, Parving HH, et al. Years of life gained by multifactorial intervention in patients with type 2 diabetes mellitus and microalbuminuria: 21 years follow-up on the Steno-2 randomised trial. Diabetologia. 2016; 59(11):2298-307. doi: 10.1007/s00125-016-4065-6.

58. Maser RE, Lenhard MJ. An overview of the effect of weight loss on cardiovascular autonomic function. Curr Diabetes Rev. 2007; 3(3): 204-11.

59. Shi L, Ye X, Lu M, Wu EQ, Sharma H, Thomason D, et al. Clinical and economic benefits associated with the achievement of both HbA1c y LDL cholesterol goals in veterans whith type 2 diabetes. Diabetes Care. 2013; 36(10): 3297-304. doi: 10.2337/dc13-0149.

60. Zoungas S, de Galan BE, Ninomiya T, Grobbee D, Hamet P, Heller S, et al. Combined effects of routine blood pressure lowering and intensive glucose control on macrovascular and microvascular outcomes in patients with type 2 diabetes: new results from the ADVANCE trial. Diabetes Care 2009, 32(11): 2068-74. doi: 10.2337/dc09-0959.

61. Zib I, Jacob AN, Lingvay I, Salinas K, McGavock JM, Raskin $\mathbf{P}$, et al. Effect of pioglitazone therapy on myocardial and hepatic steatosis in insulin-treated patients with type 2 diabetes. J Investig Med 2007; 55(5):230-6. doi: 10.2310/6650.2007.00003.

62. Holman RR, Paul SK, Bethel MA, Matthews DR, Neil HAW. 10-year follow-up of intensive glucose control in type 2 diabetes. N Engl J Med 2008; 359:1577-89. doi: 10.1056/NEJMoa0806470.

63. Low Wang CC, Hess CN, Hiatt WR, Goldfine AB. Clinical Update: Cardiovascular disease in diabetes mellitus: Atherosclerotic cardiovascular disease and heart failure in type 2 diabetes mellitus - mechanisms, management, and clinical considerations. Circulation. 2016; 133(24):2459-502. doi: 10.1161/CIRCULATIONAHA.116.022194

64. Eurich DT, Weir DL, Majumdar SR, Tsuyuki RT, Johnson JA, Tjosvold L, et al. Comparative safety and effectiveness of metformin in patients with diabetes mellitus and heart failure: systematic review of observational studies involving 34,000 pa- tients. Circ Heart Fail 2013; 6(3):395-402. doi: 10.1161/CIRCHEARTFAILURE.112.000162.

65. Kaneho M, Narukawa M. Assessment of the risk of hospitalization for heart failure with Dipeptidyl Peptidase-4 inhibitors, Saxagliptin, Alogliptin, and Sitagliptin in patients with type 2 diabetes, using an alternative measure to the hazard ratio. Ann Pharmacother. 2017; 51(7):570-6. doi: $10.1177 / 1060028017698496$

66. Chilton R, Tikkanen I, Cannon CP, Crowe S, Woerle HJ, Broedl UC, et al. Effects of empagliflozin on blood pressure and markers of arterial stiffness and vascular resistance in patients with type 2 diabetes. Diabetes Obes Metab. 2015; 17(12): 1180-93. doi: 10.1111/dom.12572.

67. Zinman B, Wanner C, Lachin JM, Fitchett D, Bluhmki E, Hantel S, et al; EMPA-REG OUTCOME Investigators. Empagliflozin, cardiovascular outcomes, and mortality in type 2 diabetes. N Engl J Med 2015; 373:2117-28.

doi: 10.1056/NEJMoa1504720.

68. Fitchett D, Zinman B, Wanner C, Lachin JM, Hantel S, Salsali A, et al; EMPA-REG OUTCOME trial investigators. Heart failure outcomes with empagliflozin in patients with type 2 diabetes at high cardiovascular risk: results of the EMPA-REG OUTCOME trial. Eur Heart J. 2016; 37(19): 1526-34. doi: 10.1093/eurheartj/ehv728.

69. Cholesterol Treatment Trialists' (CTT) Collaborators. Efficacy of cholesterol-lowering therapy in 18,686 people with diabetes in 14 randomised trials of statins: a meta-analysis. Lancet. 2008; 371(9607):117-25. doi: 10.1016/S0140-6736(08)60104-X.

70. Colhoun HM, Betteridge DJ, Durrington PN, Hitman GA, Neil HA, Livingstone SJ, et al; CARDS investigators. Primary prevention of cardiovascular disease with atorvastatin in type 2 diabetes in the Collaborative Atorvastatin Diabetes Study (CARDS): multicentre randomised placebo-controlled trial. Lancet. 2004; 364(9435):685-96. doi: 10.1016/S0140-6736(04)16895-5.

71. Sheperd J, Barter P, Carmena R, Deedwania P, Fruchart JC, Haffner S, et al. Effect of lowering LDL colesterol substantially below currently recomended levels in patients with coronary heart disease and diabetes. The Treating To New Targets (TNT) Study. Diabetes Care. 2006; 29(6): 1220-6. doi: $10.2337 / \mathrm{dc} 05-2465$.

72. Symeonides $\mathbf{P}$, Koulouris S, Vratsista E, Triantafyllou K, Ioannidis G, Thalassinos N, et al. Both ramipril and telmisartan reverse indices of early diabetic cardiomyopathy: a comparative study. Eur J Echocardiogr. 2007; 8:480-6. doi: 10.1016/j.euje.2006.09.005. 
73. Heart Outcomes Prevention Evaluation (HOPE) Study Investigators. Effects of ramipril on cardiovascular and microvascular outcomes in people with diabetes mellitus: results of the HOPE study and MICRO-HOPE substudy. Lancet 2000; 355(9200):253-9. doi: 10.1016/S0140-6736(99)12323-7.

74. Mazza A, Schiavon L, Zuin M, Lenti S, Ramazzina E, Rubello D, et al. Effects of the antihypertensive fixed-dose combinations on an early marker of hypertensive cardiac damage in subjects at low cardiovascular risk. Am J Hypertens 2016; 29(8):969-75. doi: 10.1093/ajh/hpw022. Disponible en: https://www.ncbi. nlm.nih.gov/pubmed/27053407.

75. Giannetta E, Isidori AM, Galea N, Carbone I, Mandosi E, Vizza CD, et al. Chronic inhibition of cGMP phosphodiesterase 5A improves diabetic cardiomyopathy: a randomized, controlled clinical trial using magnetic resonance imaging with myocardial tagging. Circulation 2012; 125(19):2323-33. doi: 10.1161/CIRCULATIONAHA.111.063412.

76. Kaleta B, Boguska A. Sildenafil, a phosphodiesterase type 5 inhibitor, downregulates osteopontin in human peripheral blood mononuclear cells. Arch Immunol Ther Exp 2017; 65(4):347-53. doi: 10.1007/s00005-017-0455-9.

77. Zhou X, Chen J. Is treatment with trimetazidine beneficial in patients with chronic heart failure? PLoS ONE 2014; 9(5): e94660. doi: 10.1371/journal.pone.0094660.

78. Gomes MB, Negrato CA. Alpha-lipoic acid as a pleiotropic compound with potential therapeutic use in diabetes and other chronic diseases. Diabetol Metab Syndr 2014; 6(1):80 doi: 10.1186/1758-5996-6-80

79. Heinisch BB, Francesconi M, Mittermayer F, Schaller G, Gouya G, Wolzt M, et al. Alpha-lipoic acid improves vascular endothelial function in patients with type 2 diabetes: a placebo-controlled randomized trial. Eur J Clin Invest. 2010; 40(2):148-54. doi: 10.1111/j.1365-2362.2009.02236.x.

80. Huynh K, Kiriazis H, Du XJ, Love JE, Jandeleit-Dahm KA, Forbes JM, et al. Coenzyme Q10 attenuates diastolic dysfunction, cardiomyocyte hypertrophy and cardiac fibrosis in the $\mathrm{db} / \mathrm{db}$ mouse model of type 2 diabetes. Diabetologia. 2012; 55(5): 1544-53. doi: 10.1007/s00125-012-2495-3.

81. Mortensen SA, Rosenfeldt F, Kumar A, Dolliner P, Filipiak KJ, Pella D, et al; Q-SYMBIO Study Investigators. The effect of coenzyme Q10 on morbidity and mortality in chronic heart failure: results from Q-SYMBIO: a randomized double-blind trial. JACC Heart Fail. 2014; 2(6): 641-9. doi: 10.1016/j.jchf.2014.06.008.
82. Baur JA, Pearson KJ, Price NL, Jamieson HA, Lerin C, Kalra A, et al. Resveratrol improves health and survival of mice on a high-calorie diet. Nature 2006; 444(7117): 337-42. doi: 10.1038/nature05354.

83. Timmers S, Konings E, Bilet L, Houtkooper RH, Van de Weijer T, Goossens GH, et al. Calorie restriction-like effects of 30 days of resveratrol supplementation on energy metabolism and metabolic profile in obese humans. Cell Metab. 2011; 14(5):612-22. doi: 10.1016/j.cmet.2011.10.002

84. Sulaiman M, Matta MJ, Sunderesan NR, Gupta MP, Periasamy M, Gupta M. Resveratrol, an activator of SIRT1, upregulates sarcoplasmic calcium ATPase and improves cardiac function in diabetic cardiomyopathy. Am J Physiol Heart Circ Physiol. 2010; 298(3): H833-H843. doi: 10.1152/ajpheart.00418.2009.

85. Wong RHX, Berry NM, Coates AM, Buckley JD, Bryan J, Kunz I, et al. Chronic resveratrol consumption improves brachial flow-mediated dilatation in healthy obese adults. J Hypertens. 2013; 31(9):1819-27. doi: 10.1097/HJH.0b013e328362b9d6.

86. Raj P, Louis XL, Thandapilly SJ, Movahed A, Zieroth S, Netticadan T. Potential of resveratrol in the treatment of heart failure. Life Sciences. 2014; 95(2):63-71. doi: 10.1016/j.lfs.2013.12.011.

87. Polley KR, Jenkins N, O'Connor P, McCully K. Influence of exercise training with resveratrol supplementation on skeletal muscle mitochondrial capacity. Appl Physiol Nutr Metab. 2016; 41(1):26-32. doi: 10.1139/apnm-2015-0370.

88. Evans HM, Howe PRC, Wong RHX. Effects of resveratrol on cognitive performance, mood and cerebrovascular function in post-menopausal women; A 14-week randomised placebo-controlled intervention trial. 2017 Nutrients 2017; 9 (1):27. doi: 10.3390/nu9010027.

89. Brasnyó P, Molnár GA, Mohás M, Markó L, Laczy B, Cseh J, et al. Resveratrol improves insulin sensitivity, reduces oxidative stress and activates the Akt pathway in type 2 diabetic patients. Br J Nutr 2011; 106(3):383-9. doi: 10.1017/S0007114511000316

90. Bashmakov YK, Assaad-Khalil SH, Abou Seif M, Udumyan R, Megallaa M, Rohoma KH, et al. Resveratrol promotes foot ulcer size reduction in type 2 diabetes patients. ISRN Endocrinology 2014; 2014:8p. ID 816307. doi: 10.1155/2014/816307.

91. Hausenblas HA, Schoulda JA, Smoliga JM. Resveratrol treatment as an adjunct to pharmacological management in type 2 diabetes mellitus-systematic review and meta-analysis. Mol Nutr Food Res 2015; 59(1):147-59. 